\title{
Problema de Alocação de Controladores SDN: Uma Análise do Custo em Redes de Backbone
}

\author{
Marcelo Santos ${ }^{1}$, Stênio Fernandes ${ }^{1}$ \\ ${ }^{1}$ Centro de Informática (CIn) - Universidade Federal de Pernambuco (UFPE) \\ Recife - PE - Brasil \\ \{mabs, sflf\}@cin.ufpe.br
}

\begin{abstract}
The SDN Placement Problem is an unsolved challenge, and it is considered an NP-Hard problem similarly to the set covering problems. Hence, a mathematical model based on Linear Programming is proposed to find a suitable controller placement solution to minimize the controller deployment cost. In order to validate the proposed model, the placement cost was analyzed through 112 backbone networks from the topology-zoo dataset. After that, the controller deployment cost was estimated and analyzed which topology characteristics affect the cost.
\end{abstract}

Resumo. O problema de alocação de controladores em Redes Definidas por Software (SDN) é um desafio em aberto por ser um problema de alocação NPCompleto análogo aos problemas clássicos de recobrimento de rede. Para solucionar esse problema, nós propomos um modelo matemático baseado em Programação Linear que realiza a distribuição de controladores SDN minimizando o custo de alocação de todos os controladores. Para validar nossa proposta foram estimados os custos de recobrimento de 112 redes de backbone. Em seguida foram analisadas quais características são determinantes no custo de implantação de controladores SDN de acordo com as características de cada rede.

\section{Introdução}

Redes Definidas por Software (SDN) criou um paradigma relativamente recente que vem sendo discutido dentro das reuniões da IETF/IRTF em vários work groups (WGs) e research groups (RGs), com destaque para o grupo SDNRG. Em resumo, SDN consiste em remover o plano de controle de cada switch e colocá-los em uma entidade externa e centralizada (ou seja, um controlador SDN) a fim de permitir novas funcionalidades, tais como, por exemplo, manipular fluxos de rede através de uma interface programável. Consequentemente há uma visão global da rede pelo controlador possibilitando atender novas demandas tanto por parte dos usuários quanto provedores de infraestrutura. Além disso, SDN é vista como uma das tecnologias que vai servir como base para a Internet do Futuro, buscando ajudar a superar barreiras como a ossificação da Internet (Esteves, Granville, \& Boutaba, 2013). Nesse contexto, juntamente com seus benéficos, emergem diversos desafios de otimização que vem sendo atacados pela indústria e pela academia, como por exemplo: (1) Problema de alocação de controladores SDN; (2) Problemas de alocação de regras de controladores SDN e (3) Problemas de alocação de Hypevisors SDN. 
Decidir qual o tipo de controlador SDN, quais protocolos devem ser utilizados, a capacidade ideal de cada controlador, bem como a localização destes novos controladores são questões fundamentais que influenciam diretamente aspectos como desempenho, custo, resiliência, tempo de instalação de regras nos switches, tempo de convergência entre controladores e sua capacidade de escalabilidade. Tais questões foram discutidas pelos autores na reunião 95 da IETF/IRTF realizada em Buenos Aires na Argentina no grupo SDNRG ${ }^{1}$. Assim, este artigo tem como uma de suas contribuições estender tal discussão na comunidade brasileira do IETF /IRTF com ênfase na análise do problema de alocação de controladores SDN.

Um controlador SDN é um ponto estratégico importante de controle na rede. Decidir onde alocar os controladores SDN, quais switches são controlados por cada controlador SDN (estimando sua capacidade) e quantos controladores são necessários para que não haja um ponto limitante são questões importantes a fim de minimizar o custo de recobrimento da rede e garantir um bom desempenho. Note que a disposição dos controlares SDN pode afetar o tempo de configuração de um novo fluxo que chega a um switch. Consequentemente, devido a restrições de tempo de comunicação mínimo entre controlador e switch, em alguns casos, torna-se impossível cobrir toda a rede com um único controlador, mesmo que um controlador possuía capacidade suficiente para controlar toda a rede. Seguindo esse raciocínio, decidimos investigar redes de backbone levantado questões como: (1) quais características de uma rede de backbone podem influenciar no custo da implantação de controlares de uma rede SDN? (2) como as restrições de tempo de comunicação podem afetar o custo e a quantidade final de controladores? (3) qual a relação entre tamanho da topologia e o custo de implantação dos controladores? e (4) qual a relação entre o atraso dos enlaces da rede e o custo de implantação dos controladores?

Nesse contexto, abordamos o problema de alocação de controladores SDN sob a perspectiva do custo financeiro de implantação considerando que existem capacidades variáveis do controlador. O primeiro passo da solução é baseado no uso de programação linear. A partir da modelagem realizada utilizamos a ferramenta CPLEX ${ }^{2}$ da IBM para analisar topologias de rede reais fornecidas pelo Topology-Zoo (Knight, Nguyen, Falkner, Bowden, \& Roughan, 2011) e determinar a melhor alocação de controladores para cada rede. Dessa forma, as contribuições deste artigo são três: (1) um modelo formal para o problema de alocação de controladores SDN que minimiza os custos de implantação; (2) Investigação de quais características de topologias afetam o custo de alocação de controladores SDN e (3) Discussão de propostas de drafts para o SDNRG.

Nosso modelo pode ser usado, por exemplo, quando um provedor de infraestrutura decide migrar para uma solução SDN. Nesse cenário é necessário determinar qual conjunto de switches será atribuído a cada controlador SDN (domínio do controlador), respeitando as restrições de capacidade e o tempo de configuração de fluxo (atraso entre os switches até o controlador). Portanto, na perspectiva de provedor de infraestrutura, é possível definir este problema como um problema de alocação que busca determinar o número de controladores SDN, capacidade de cada controlador, localização na rede de cada controlador e conjunto de switches controlados de forma a minimizar o custo total de implantação.

\footnotetext{
${ }^{1}$ https://www.ietf.org/proceedings/95/slides/slides-95-sdnrg-2.pdf

${ }^{2} \mathrm{http}: / / \mathrm{www}-01$. ibm.com/software/commerce/optimization/cplex-optimizer/
} 


\section{Referencial Teórico}

\subsection{Redes Definidas por Software: Conceitos Básicos}

SDN surgiu há alguns anos como uma alternativa para superar problemas enfrentados no ambiente de redes de computadores. A grande inovação baseia-se na dissociação do plano de controle do plano de dados. Dessa forma, switches tem seu plano de dados gerenciado por um controlador SDN (entidade que agem como o plano de controle). Consequentemente, ao contrário das redes tradicionais, esses switches não implementam o plano de controle e as decisões de encaminhamento são decididas por fluxos encaminhados do switch para o controlador.

Um plano de controle centralizado num controlador SDN permite uma visão única e global da rede, trazendo benefícios como um conjunto de políticas menos propensas a erros, configurações de dispositivos de baixo nível mais simples e adaptações dinâmica da rede. O controlador SDN, ou eventuais aplicações executadas sobre o controlador, são responsáveis por atualizar a tabela de encaminhamento de cada roteador/switch da rede. Essa abordagem permite que novas funcionalidades sejam implementadas simplesmente pela adição de novas peças de software (aplicações) que realizam o gerenciamento da rede através do controlador SDN. Consequentemente, uma rede SDN tem uma camada de abstração que permite a implementação de uma variedade de algoritmos como balanceamento e classificação de tráfego com base em restrições definidas pelo operador de rede. Portanto, é comum se referir a controladores SDN como o sistema operacional de rede (Nunes et al., 2014).

Note que quando um switch não possui uma regra para encaminhar determinado pacote, o switch deve encaminhar o pacote para seu controlador que baseado em alguma aplicação de gerenciamento decidirá qual o melhor caminho para rotear este fluxo novo na rede. Por esta razão, o tempo de comunicação (atraso) entre o controlador e switches pode ser um fator importante no desempenho de uma rede SDN. Atualmente, a comunicação entre controladores SDN e dispositivos de encaminhamento é realizada principalmente através do protocolo OpenFlow (Jarraya, Madi, \& Debbabi, 2014). Uma boa referência para aprofundamento sobre SDN é a survey intitulada: "Software-Defined Networking: A Comprehensive Survey" (Kreutz et al., 2015).

\subsection{Trabalhos Relacionados}

Há várias propostas da academia e indústria sobre arquiteturas distribuídas de controladores SDN bem como discussões sobre técnicas de alocação. É consenso na comunidade científica que os controladores SDN devem ser distribuídos, ao invés de se ter apenas um único controlador para toda a rede. A ideia por trás da distribuição de controladores é obter maior escalabilidade, espalhar pontos de falha tanto quanto possível e reduzir o atraso entre o controlador e switches, como discutido em (Bari et al., 2013; Curtis et al., 2011; Hassas Yeganeh e Ganjali, 2012; Phemius, Bouet e Leguay, 2014).

Heller, Sherwood, \& McKeown (2012) investigaram o impacto da latência entre controladores SDN e switches de acordo com a posição dos controladores na rede. No entanto, este artigo não propõe nenhum algoritmo ou técnica de otimização aplicada para o mapeamento de controladores da SDN. Os autores testam exaustivamente todas as possíveis soluções, assumindo que precisam alocar um número fixo de controladores em uma topologia específica. Os resultados indicam que controladores bem distribuídos 
podem minimizar a latência entre os switches e controladores. Os autores não levam em conta características importantes, tais como a capacidade do controlador, balanceamento de carga e o custo de cada controlador.

Krishnamurthy, Chandrabose, \& Gember-Jacobson (2014) criam uma estratégia para atribuir switches e aplicações SDN para um determinado controlador SDN, buscando minimizar a comunicação entre controladores e o consumo de recursos. Os autores reduzem o consumo de memória RAM e de CPU em um controlador através do gerenciamento de aplicativos que são executados. Consequentemente, pode haver uma redução de custo devido ao uso de controladores de menor capacidade. Em resumo, a proposta é reduzir o consumo de recursos em cada controlador (CPU e memória RAM), além de tentar minimizar o tempo de configuração de fluxo.

Xiao et al. (2014) propuseram um algoritmo de alocação baseado em clusterização afim de determinar quantos controladores SDN são necessários para cobrir uma topologia e a posição de cada controlador. Neste trabalho, Xiao et al. não levam em consideração a heterogeneidade dos controladores com diferentes capacidades e os custos financeiros envolvidos. Além disso, a avaliação da solução proposta foi realizada em uma única topologia, que limita a validação da solução.

\section{Uma solução ótima para minimização do custo de controladores SDN através do uso de Programação Linear (PL)}

Nós modelamos o problema de alocação de controladores SDN usando programação linear inteira (PLI) com o objetivo de minimizar o custo final de alocação de todos os controladores de forma que todos os switches sejam cobertos por pelo menos um controlador. Programação linear permite obter soluções ótimas geralmente com um alto custo computacional quando temos um problema NP-difícil (Lange et al., 2015) com muitas variáveis envolvidas. Em problemas de cobertura de redes, como o apresentado neste trabalho, é computacionalmente dispendioso encontrar uma solução ótima diante de redes com um grande número de nós.

Em nosso modelo o número máximo de controladores SDN que podem ser alocados é igual ao número de switches da rede. Assumimos que cada controlador adicionado é obrigatoriamente ligado a um switch existe na rede. Em outras palavras, cada switch da rede pode ser visto como um potencial ponto de alocação de um controlador. A seguir temos uma descrição do modelo:

A) Notação e constantes:

$$
\begin{aligned}
& N=\text { Número de nós (switches) } \\
& M=\text { Um número grande (Constante) } \\
& S_{i}=\text { número de fluxos gerado por um switch } i \\
& A=\text { conjunto de arcos existentes }(i, j) \\
& D_{i j}=\text { atraso do enlace entre os nós }(i, j) \\
& K=\text { quatidade de tipos de controladres } S D N \text { (índice } k \text { ) } \\
& F_{k}=\text { custo de um controlador do tipo } k \\
& C_{k}=\text { capacidade de um controlador do tipo } k \\
& \beta=\text { restrição de atraso entre um controlador e um switch } \\
& \delta=\text { restrição de atraso entre controladores }
\end{aligned}
$$

B) Variáveis de decisão: 


$$
\begin{aligned}
& y_{i j}=\left\{\begin{array}{c}
1, \text { se um nó } i \text { é atribuído a um controlador } j \\
0, \quad \text { caso controário }
\end{array}\right. \\
& x_{k j}=\left\{\begin{array}{c}
1, \text { se ocontrolador do tipo } k \text { é alocado em } j \\
0, \quad \text { caso controário }
\end{array}\right.
\end{aligned}
$$

C) Função Objetivo:

A função objetivo é definida como a minimização do custo de cobertura da rede por controladores SDN considerando que podem haver diferentes tipos de controladores. Consequentemente, cada controlador tem uma capacidade e um custo específico:

$$
\operatorname{Min} \sum_{k=1}^{K} \sum_{j=1}^{M} x_{k j} F_{k}
$$

D) Restrições:

Equação 2 garante que todo switch é associado a um controlador alocado em $j$ :

$$
\sum_{j=1}^{N} y_{i j}=1, \quad \forall i
$$

Considerando que cada controlador tem uma capacidade e custo diferente, temos uma restrição que permite existir apenas um controlador alocado em $j$ :

$$
\sum_{k=1}^{K} x_{k j} \leq 1, \quad \forall j
$$

Nós assumimos que um switch $i$ gera um número de fluxos $S_{i}$. Desta forma, a capacidade de um controlador SDN deve ser igual ou maior a soma de todos os fluxos gerados pelos switches que lhe foram atribuídos:

$$
\sum_{j=1}^{N} y_{i j} S_{i} \leq \sum_{k=1}^{K} x_{k j} C_{k}, \quad \forall i
$$

Como exemplo, se todos os switches gerarem $10 \mathrm{mil}$ fluxos/s $\left(S_{i}=10 \mathrm{~K}\right)$ e um controlador tiver a capacidade para lidar com $1000 \mathrm{mil}$ fluxos/s, logo esse controlador pode suportar no máximo 100 switches.

Para garantir que haja um atraso máximo entre um switch $i$ e um controlador alocado na posição $j$, temos:

$$
y_{i j} D_{i j} \leq \beta, \quad \forall(i, j) \in A
$$

A equação (6) garante um atraso máximo entre quaisquer controladores da rede alocados numa posição $i$ e $j$. Note que $D_{i j}$ é uma matriz de atraso entre todos os nós da topologia e é uma das entradas da simulação. 


$$
\sum_{k=1}^{K} x_{k j} \cdot D_{i j} \leq \delta+\left(1-\sum_{k=1}^{K} x_{k i}\right) M, \quad(i, j) \in A
$$

Condições de integralidade e não negatividade:

$$
\begin{aligned}
& x_{k j} \in\{0,1\} \quad \forall i, j \\
& y_{i j} \in\{0,1\} \quad \forall i, j
\end{aligned}
$$

\section{Metodologia de Avaliação}

\subsection{Topologia}

Foram analisadas 112 topologias do Topology-Zoo (Knight et al., 2011) aplicando o modelo exibido na seção 4. Anomalias foram removidas em algumas topologias como, por exemplo, os nós com grau zero (sem enlaces). Algumas das topologias disponíveis têm mais de uma versão, nós usamos somente as versões mais recentes para não viesar os resultados.

Através da localização geográfica de nós, estimamos o atraso entre os diferentes nós da topologia. As Equações de 9 e 10 foram usadas para calcular a distância entre os nós e o atraso.

$$
D=R * \arccos \left(\begin{array}{c}
\sin (a) \sin (c)+ \\
\cos (a) * \cos (c) * \cos (b-d)
\end{array}\right)
$$

D é a distância em Km; R é igual a 6371 (raio médio da terra); (a, b) é a latitude e a longitude do switch i e (c, d) é a latitude e a longitude do switch j. Assim, o atraso é estimado através da seguinte equação (JIN \& JIN, 2009):

$$
R T T=P * D
$$

$\mathrm{P}$ é uma métrica relacionada à condição de rede, segundo (JIN e JIN, 2009), P = $20 \mathrm{us} / \mathrm{km}$. Quando a latitude e longitude não estão disponíveis em alguns nós das topologias do Topology-zoo, assumimos que o atraso do enlace é de $15 \mathrm{~ms}$. A matriz de caminho mais curto entre todos os nós é obtida usando a biblioteca JGraphT $^{3}$ e o algoritmo de Dijkstra. Esta matriz é usada como entrada do modelo para determinar o atraso entre cada controlador SDN e os switches que compõe a rede. Para solucionar o problema de programação linear modelado foi utilizado o CPLEX da IBM (Cplex, 2007).

\subsection{Custo e Capacidade do Controlador SDN}

Grandes fornecedores de dispositivos de rede, como HP, Alcatel, Juniper e VMware entraram forte no mercado de SDN fornecendo switches OpenFlow, controladores SDN ou soluções completas de SDN. O custo atrelado a uma migração para uma rede SDN exige um custo inicial de investimento (CAPEX - despesas de capital) devido à grande mudança realizada na rede. Incidindo sobre o custo do controlador SDN podemos destacar duas categorias importantes: (1) hardware e software (2).

Estimar o custo de um controlador é uma tarefa difícil devido a vários atributos que podem compor esse valor. Há diversas opções de software gratuitos para

\footnotetext{
${ }^{3}$ JGraphT - http://jgrapht.org/
} 
controladores SDN tais como NOX, $\mathrm{POX}^{4}$, Floodlight ${ }^{5}$ e OpenDayLight ${ }^{6}$, bem como soluções proprietárias como o controlador HP VAN SDN. O problema relacionado a controladores gratuitos é a falta de suporte e atualizações. Por outro lado, uma solução proprietária como a oferecida pela HP possui custos variáveis de acordo com a quantidade de switches gerenciados pelo controlador. Ou seja, à medida que aumenta o tamanho da rede, o custo também sobe. A HP recomenda que os requisitos mínimos do sistema sejam: 16 GB de RAM; 64 GB de HD e adaptador de rede Ethernet Gigabit que acarretam custos de hardware7. Obviamente, o desempenho do controlador SDN é dependente da capacidade do hardware subjacente que está sendo utilizado. Assim, é razoável estimar que o controlador SDN custa alguns milhares de reais (custo de hardware e software).

O desempenho do controlador varia de acordo com a combinação de hardware e software. Por conseguinte, nós optamos por usar único número para representar todos os custos atribuídos a um controlador. Shah et al. (2013) mostra que dentre os controladores avaliados a capacidade de manipular fluxos varia de 1 a 4 milhões de fluxos/s. Nós assumimos que é possível optar por um controlador que possua uma menor capacidade e um menor custo ao invés de considerar todos os controladores com uma única capacidade e custo, possibilitando assim uma maior capacidade de otimização de custos. Podemos ver na Tabela 1 a capacidade do controlador representada como a quantidade de fluxos que o controlador pode manipular e o seu respectivo custo em reais. Note que tais entradas no modelo são facilmente modificadas para representar custo e capacidade de controladores atendendo a outros cenários.

\subsection{Tempo de Configuração do Fluxo (Flow setup time) e Geração de Fluxos}

Quando um switch recebe um novo fluxo e não possui uma regra de como encaminhá-lo, deve-se então requisitar ao controlador uma regra de encaminhamento adequada de acordo com o que o controlador considera mais adequado. O tempo requerido para executar essa operação é conhecido como tempo de configuração do fluxo (flow setup time). Um flow setup time de $10 \mathrm{~ms}$ pode adicionar um atraso de $10 \%$ na comunicação em fluxos de curta de duração (Tootoonchian, Gorbunov, Ganjali, Casado, \& Sherwood, 2012). Sendo assim, um fator importante a ser analisado.

Segundo Benson et al. (Benson, Akella, \& Maltz, 2010), um conjunto de 100 switches pode gerar picos de mais de 10 milhões de fluxos/s considerando um cenário de congestionamento da rede. Consequentemente, nós consideramos aceitável estimar que cada switch em nossa simulação gerasse uma carga de 70.000 fluxos/s sobre o controlador ao qual foi atribuído.

Pensando ainda no tempo de sincronização entre controladores e na convergência sobre as decisões de encaminhamento tomadas, definimos uma restrição de atraso máximo de 200ms (Bari, Roy, Chowdhury, \& Zhang, 2013) entre todos os controladores alocados em uma mesma topologia. Assim, podemos ver nas Tabelas 1 e 2 os valores de todas as restrições assumidas em relação ao flow setup time, atraso entre controladores, parâmetros, fatores e níveis.

\footnotetext{
${ }^{4}$ openflow.stanford.edu/display/ONL/POX+Wiki

${ }^{5} \mathrm{http}$ ://www.projectfloodlight.org/

${ }^{6}$ www.opendaylight.org

${ }^{7} \mathrm{http} / / / \mathrm{h} 20564$.www2.hpe.com/hpsc/doc/public/display?docId=emr_na-c03967703
} 


\subsection{Fatores, Níveis e Entradas do Modelo}

Efetuamos vários experimentos de acordo com as diferentes características e parâmetros apresentados na Tabela 1 e Tabela 2. Variamos a restrição de atraso entre um switch e um controlador, além de considerar uma restrição de atraso entre controladores.

Tabela 1. Fatores e Níveis

\begin{tabular}{|c|c|c|c|}
\hline Fatores & Unidade & Níveis & Valores \\
\hline $\begin{array}{c}\text { Restrição entre switch e } \\
\text { controlador }\end{array}$ & $\mathrm{ms}$ & 8 & $25,50,75,100,125,150,175,200$ \\
\hline $\begin{array}{c}\text { Topologia } \\
\text { (backbone) }\end{array}$ & ---- & 112 & Redes do Topology-Zoo' \\
\hline Capacidade do Controlador & Fluxos (M) & 3 & 1,2 or 4 \\
\hline Custo do Controlador & Reais (R\$) & 3 & $10.000,15.000$ e 20.000 \\
\hline
\end{tabular}

Tabela 2. Parâmetros

\begin{tabular}{|c|c|}
\hline Parâmetro & Valor \\
\hline Fluxos gerados por um switch j $\left(S_{j}\right)$ & 70K (fluxos) \\
\hline Atraso dos enlaces & Definido pela equação 9 and 10 \\
\hline Restrição entre controladores & 200ms \\
\hline
\end{tabular}

Em essência, as entradas são: (1) topologia composta por nós, enlaces e atraso dos enlaces e (2) Especificação de características como o atraso entre controladores e switch, capacidade que um controlador pode manipular fluxos oriundos de um conjunto de switches e taxa de geração de fluxos de um switch. Como saída de modelo, se houver pelo menos uma solução válida, temos a saída da resolução do problema de recobrimento de uma rede de backbone para uma rede SDN. O modelo de programação linear foi expresso em Java e resolvido pelo software CPLEX versão 12.61.

Os experimentos foram realizados em uma máquina com as seguintes configurações: Intel ${ }^{\circledR}$ Core $^{\mathrm{TM}}$ i7-4500U CPU @1.8GHz; 8GB RAM; 1TB HD e Sistema Operacional Windows 10 Professional 64bits. Aproximadamente 900 experimentos foram executados.

\section{Resultados e Discussão}

Nesta seção, vamos apresentar e comparar os resultados da solução proposta. Como dito anteriormente, nós executamos diferentes combinações de fatores e níveis, com o objetivo de compreender o impacto no custo de implantação de uma rede SDN.

\subsection{Como a restrição de atraso entre controladores SDN e switches afetam o custo de alocação?}

Na Figura 1 temos as médias das topologias analisadas. As barras representam o número médio de controladores necessários para cobrir as topologias analisadas. Já a linha tracejada representa o custo médio desses controladores. O intervalo de confiança de $95 \%$ foi calculado e exibido na mesma figura.

Podemos notar que a variação da restrição de atraso entre o controlador SDN e os switches pertencentes ao seu domínio não teve um grande impacto no número de controladores SDN e consequentemente no custo de implantação dos controladores. Com exceção para a restrição de $25 \mathrm{~ms}$ e $50 \mathrm{~ms}$ que reduzem a capacidade de um controlador 
alcançar um switch que esteja geograficamente mais distante mesmo que tal controlador possua capacidade suficiente para manipular mais fluxos de redes. Vemos na Figura 1 que ao considerar uma restrição de $50 \mathrm{~ms}$ ou maior 2 controladores são, em média, suficientes para cobrir toda a rede com um custo entre 20 e 25 mil reais. Nota-se uma estabilização do custo e número de controladores SDN quando a restrição de atraso entre controlador e switch é maior ou igual a 50ms. Este comportamento se deve às características intrínsecas das topologias avaliadas que possuem baixo atraso entre seus nós. Em outras palavras, como o atraso entre os nós é baixo, a restrição de atraso não é um fator de grande impacto no custo durante o processo de alocação.

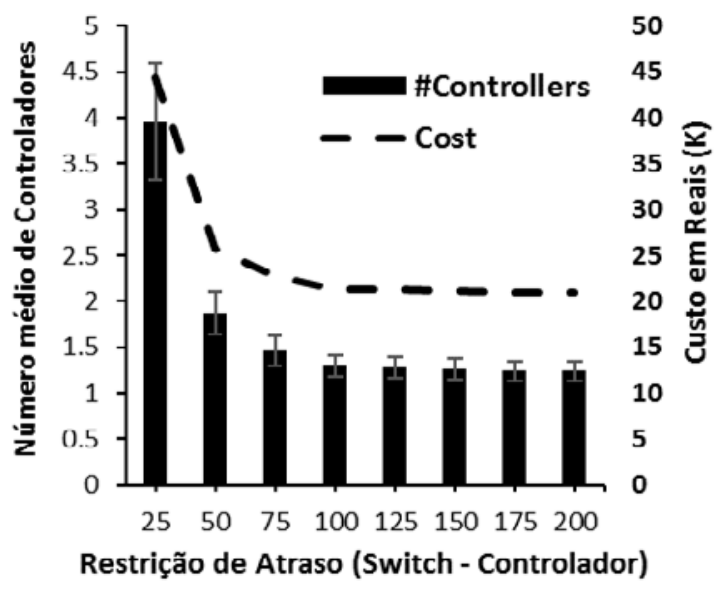

Figura 1. Custo e Número de controladores SDN



Figura 2. Casos sem solução viável encontrados

Devido às restrições para alocação dos controladores nem sempre foi possível encontrar uma solução viável. Obviamente que quanto mais rígida a restrição, o espaço de soluções viáveis tende a ser menor. Assim, podemos ver na Figura 2 que dentre as 112 topologias analisadas considerando uma restrição de $25 \mathrm{~ms}$ entre o controlador e o switch temos 19 casos em que não conseguimos encontrar nenhuma solução viável. Quando relaxamos essa restrição para $200 \mathrm{~ms}$ o número de casos cai para 8 . Cabe ressaltar que a restrição de atraso entre todos os controladores foi sempre de $200 \mathrm{~ms}$ em todas as simulações executadas. Dessa forma, quando temos tais restrições atuando em conjunto torna-se impossível em certos casos encontrar uma solução devido ao grande número de nós da topologia e o alto atraso entre seus nós.

É importante salientar que embora a média de controladores necessários para cobrir uma rede seja próximo de dois, não necessariamente todas as topologias são satisfeitas por 2 controladores que possuem a mesma capacidade ou até mesmo que apenas 2 controladores seja suficiente. Por isso, analisamos nas subseções abaixo a correlação entre: (1) custo $\mathrm{x}$ tamanho da topologia e (2) custo $\mathrm{x}$ atraso médio entre os nós que compõe a topologia.

\subsection{Qual a correção entre o custo e atraso com a média dos enlaces que compõe uma topologia?}

Uma das questões que surgem quando consideramos restrições de atraso entre controlador e switch e entre os próprios controladores são quais características da topologia podem influenciar no custo de alocação. Para esclarecer tal questão, analisamos a correlação 
entre o atraso médio dos enlaces de uma topologia e o custo final de alocação dos controladores SDN em nossos experimentos. Podemos ver na Figura 3 que a correlação do custo com o atraso médio dos enlaces de uma topologia é fraca.

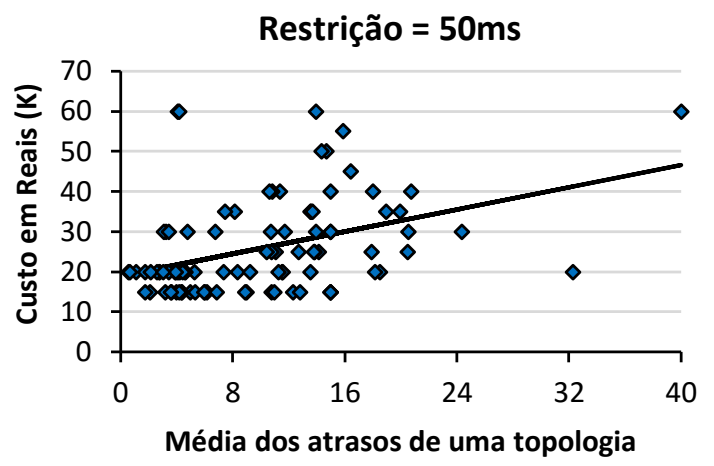

(a)

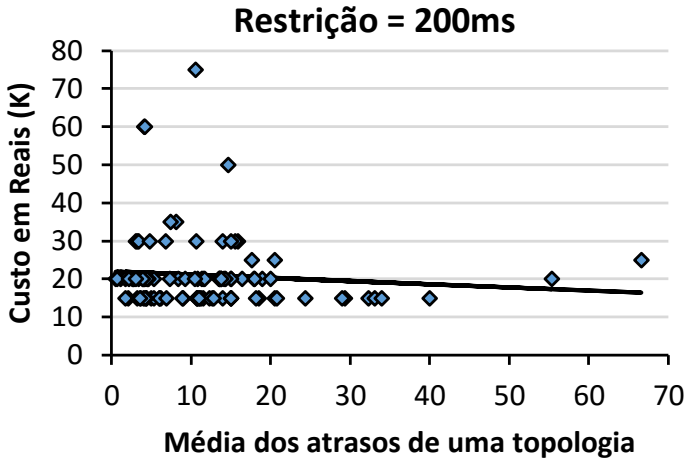

(b)

Figura 3. Correlação entre custo e atraso médio de uma topologia

$\mathrm{Na}$ figura 4(a) a correlação foi de apenas 0.4 contra 0.09 na Figura 4(b). No geral a correlação variou de 0.09 a 0.56 considerando as diferentes restrições entre switch e controlador consideradas. Por conseguinte, podemos afirmar que não há uma correlação forte entre custo e atraso dos enlaces das topologias analisadas.

\subsection{Qual a correlação entre o custo e o número de nós de nós que compõe uma topologia?}

Analisando a correlação entre o tamanho da topologia e o custo, nós estamos investigando basicamente a quantidade de controladores exigidos para cobrir uma rede de acordo com a quantidade de switches controlados por cada controlador. Em outras palavras, se um controlador não possui capacidade suficiente para manipular a quantidade de fluxos gerados pela quantidade de switches em seu domínio, será então necessário a implantação de outro controlador que acarretará um maior custo.

Podemos ver na Figura 4 há uma correlação forte entre essas métricas. Na figura 5(a) temos uma correlação de 0.68 e na Figura 5(b) uma correlação de 0.97 . No geral a correlação variou de 0.38 a 0.97 considerando as diferentes restrições entre switch e controlador consideradas. Por conseguinte, podemos afirmar que há uma correlação forte entre custo e atraso dos enlaces das topologias analisadas influenciada pela restrição de atraso defina entre do controlador e switch.

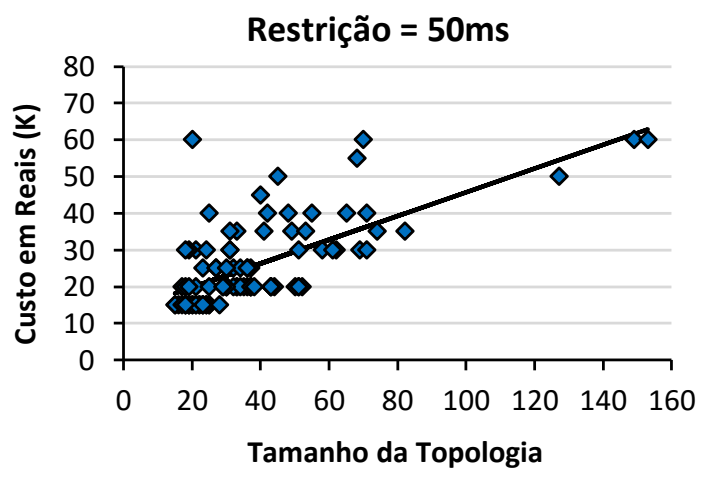

(a)

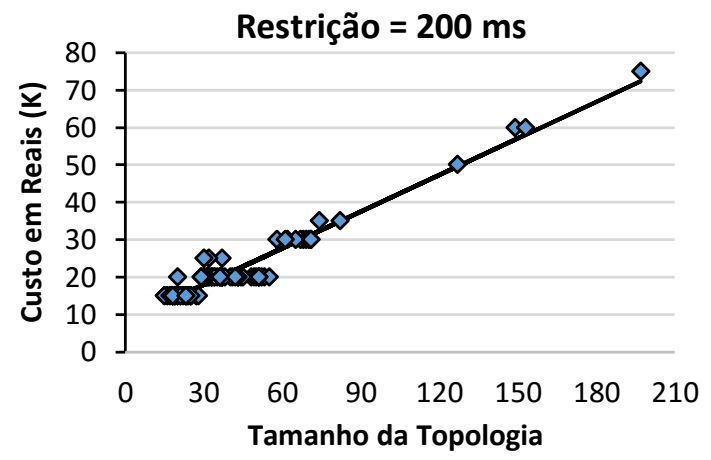

(b)

Figura 4. Correlação entre custo e tamanho da topologia 
O fato da correlação ser menor quando a restrição de atraso é mais rígida (Figura 4a) entre controlador e switch deve-se ao fato do controlador não poder cobrir um número maior de switches devido ao atraso e não necessariamente pela capacidade do controlador.

\section{Conclusão e Trabalhos Futuros}

Neste artigo apresentamos uma solução para o problema de alocação de controladores SDN onde o objetivo é minimizar o custo financeiro de migração de uma rede tradicional para uma rede SDN. Uma solução ótima é obtida utilizando programação linear. Analisamos o modelo proposto em 112 topologias reais classificadas como redes de backbone. Através dos resultados obtidos realizamos uma análise de quais características influenciaram no custo de final de alocação dos controladores.

Os resultados revelam que em média dois controladores são suficientes para cobrir uma rede classificada como backbone. Vimos que devido as características das topologias analisadas a restrição de atraso entre controlador e switch provocou pouco impacto no custo de alocação dos controladores SDN. Analisando a correlação de algumas métricas chegamos à conclusão de que há uma fraca correlação entre o custo e a média do atraso dos enlaces de uma topologia do Topology-Zoo. Por outro lado, identificamos uma tendência de correlação entre o tamanho da topologia e o custo de alocação dos controladores, chegando em determinados cenários a atingir uma correlação de 0.97.

Possíveis drafts podem surgir do trabalho realizado como, por exemplo, um informational draft sobre os tipos de problema de alocação em SDN (Alocação de Controladores, Alocação de Regras e Alocação de Hypervisors), suas características e desafios. Outro informational draft que pode surgir desse trabalho diz respeito as técnicas para resolução desses problemas e suas respectivas vantagens e desvantagens. De formal geral, o trabalho desenvolvido serve como guia sobre quais características são importantes em uma topologia a ponto de afetar o custo de implantação de controladores $\mathrm{SDN}$, bem como quais possíveis restrições podem acarretar um custo maior nesse processo.

Cabe ressaltar que este é um problema NP-difícil, e devido à sua complexidade, algumas topologias com muitos nós são impossíveis de serem analisadas por um método exato como programação linear devido a quantidade de tempo e recursos computacionais exigidos. Portanto, como trabalho futuro pretende-se atacar o problema através do uso de uma meta-heurística. Além disso, novas restrições deverão ser consideradas como, por exemplo, a consideração de atributos de dependabilidade.

\section{Referências}

Bari, M., Roy, A., Chowdhury, S., \& Zhang, Q. (2013). Dynamic Controller Provisioning in Software Defined Networks. International Conference on Network and Service Management.

Benson, T., Akella, A., \& Maltz, D. A. (2010). Network traffic characteristics of data centers in the wild. In Proceedings of the 10th annual conference on Internet measurement - IMC '10 (p. 267). New York, New York, USA: ACM Press.

Esteves, R. P., Granville, L. Z., \& Boutaba, R. (2013). On the management of virtual networks. IEEE Communications Magazine, 51(7), 80-88.

Heller, B., Sherwood, R., \& McKeown, N. (2012). The controller placement problem. 
ACM SIGCOMM Computer Communication Review, 42(4), 473.

Jarraya, Y., Madi, T., \& Debbabi, M. (2014). A Survey and a Layered Taxonomy of Software-Defined Networking. IEEE Communications Surveys \& Tutorials, 16(1), 11.

JIN, T., \& JIN, H. (2009). Network Delay Model for Overlay Network Application. International Journal of Communications, Network and System Sciences, 02(05), 400406.

Knight, S., Nguyen, H. X., Falkner, N., Bowden, R., \& Roughan, M. (2011). The internet topology zoo. Selected Areas in Communications, IEEE Journal on, 29(9), 1765-1775.

Kreutz, D., Ramos, F. M. V., Verissimo, P. E., Rothenberg, C. E., Azodolmolky, S., \& Uhlig, S. (2015). Software-Defined Networking: A Comprehensive Survey. Proceedings of the IEEE, 103(1), 14-76.

Krishnamurthy, A., Chandrabose, S. P., \& Gember-Jacobson, A. (2014). Pratyaastha: an efficient elastic distributed SDN control plane. In Proceedings of the third workshop on Hot topics in software defined networking - HotSDN '14 (pp. 133-138). New York, New York, USA: ACM Press.

Lange, S., Gebert, S., Zinner, T., Tran-Gia, P., Hock, D., Jarschel, M., ... Cho, H. (2015). Heuristic Approaches to the Controller Placement Problem in Large Scale SDN Networks. IEEE Transactions on Network and Service Management, 12(1), 4-17.

Nunes, B. A. A., Mendonca, M., Nguyen, X., Obraczka, K., \& Turletti, T. (2014). A Survey of Software-Defined Networking: Past, Present, and Future of Programmable Networks. IEEE Communications Surveys \& Tutorials, 16(3), 1617-1634.

Shah, S. A., Faiz, J., Farooq, M., Shafi, A., \& Mehdi, S. A. (2013). An architectural evaluation of SDN controllers. In 2013 IEEE International Conference on Communications (ICC) (pp. 3504-3508). IEEE.

Tootoonchian, A., Gorbunov, S., Ganjali, Y., Casado, M., \& Sherwood, R. (2012). On controller performance in software-defined networks. Proceeding Hot-ICE'12 Proceedings of the 2nd USENIX Conference on Hot Topics in Management of Internet, Cloud, and Enterprise Networks and Services, 10-10. Retrieved from https://www.usenix.org/system/files/conference/hot-ice12/hotice12-final33_0.pdf

Xiao, P., Qu, W., Qi, H., Li, Z., \& Xu, Y. (2014). The SDN controller placement problem for WAN. In 2014 IEEE/CIC International Conference on Communications in China (ICCC) (pp. 220-224).

\section{Agradecimentos}

Os autores gostariam de agradecer a FACEPE (IBPG-1321-1.03/11 para Marcelo Santos), CAPES e CNPq (206410/2014-0, 304422/2013-4, 482831/2012-0 para Stenio Fernandes). Por fim, gostaríamos de agradecer ao NIC.BR e ao CGI pelo suporte na participação das reuniões da IETF/IRTF. 